\title{
Retrospective Analysis of an Insulin-to-Liraglutide Switch in Patients with Type 2 Diabetes Mellitus
}

Eveline Bruinstroop (D) - Laura Meyer - Catherine B. Brouwer •

Diana E. van Rooijen · P. Sytze van Dam

Received: April 23, 2018 / Published online: May 19, 2018

(C) The Author(s) 2018

\section{ABSTRACT}

Introduction: Insulin and the GLP-1 receptor agonist liraglutide are both effective in reaching glycemic targets. The efficacy of an insulin-toliraglutide switch in an obese population with concurrent use of sulfonylurea and metformin is unknown. We assessed the efficacy and determinants of success of an insulin-to-liraglutide switch in these patients.

Methods: In a retrospective study we analyzed all patients that underwent an insulin-to-liraglutide switch during routine medical care (January 2009-February 2015). It was assessed if patients still continued liraglutide 12 months

Eveline Bruinstroop and Laura Meyer have contributed equally.

Enhanced digital features To view enhanced digital features for this article go to https://doi.org/10.6084/ m9.figshare.6200726.

Electronic Supplementary Material The online version of this article (https://doi.org/10.1007/s13300018-0438-9) contains supplementary material, which is available to authorized users.

E. Bruinstroop $(\varangle) \cdot$ L. Meyer · C. B. Brouwer .

P. S. van Dam

Department of Internal Medicine, OLVG,

Oosterpark 9, 1091 AC Amsterdam, The Netherlands

e-mail: e.bruinstroop@amc.uva.nl

D. E. van Rooijen

Department of Research and Epidemiology, OLVG,

Oosterpark 9, 1091 AC Amsterdam, The Netherlands after the switch or discontinued because of poor glycemic control or side effects. Baseline characteristics were compared between the groups to establish determinants of success.

Results: A total of 104 patients made an insulin-to-liraglutide switch (43\% male; mean age $57.2 \pm 9.9$ years; mean BMI $39.8 \pm 5.4 \mathrm{~kg} / \mathrm{m}^{2}$ ). Sixty patients still continued liraglutide after 12 months (58\%) whereas 37 patients discontinued treatment because of poor glycemic control within 12 months (36\%) and seven patients discontinued liraglutide because of intolerable side effects (7\%). Insulin dose and insulin frequency at baseline were significantly lower in patients that continued liraglutide. Patients reaching $\mathrm{HbA} 1 \mathrm{c} \leq 7 \%(53 \mathrm{mmol} / \mathrm{mol})$ showed lower baseline HbA1c levels, shorter duration of diabetes, and shorter duration of insulin therapy.

Conclusion: The majority of patients continued liraglutide after a switch from insulin therapy with on average no change in glycemic control and decrease of body weight. HbA1c levels at baseline, duration of insulin therapy, and duration of diabetes were predictive of reaching glycemic control on liraglutide alone. In current practice this also indicates which patients on insulin can reduce their insulin dose after adding a GLP-1 receptor agonist.

Plain Language Summary: Plain language summary available for this article. 
Keywords: C-peptide; GLP-1; GLP-1 receptor; Glucagon-like peptide-1; HbA1c; Hypoglycemic agent; Insulins; Insulin sensitivity; Metformin; Sulfonylurea compounds

\section{PLAIN LANGUAGE SUMMARY}

Patients with type 2 diabetes suffer from high blood glucose levels, which cause symptoms as fatigue and headaches and long-term complications such as eye disease. Therefore medication is prescribed when lifestyle measures alone cannot lower glucose levels sufficiently. The first choice of treatment is glucose-lowering pills. When glucose levels are still too high there are several options for injecting medication with a small needle under the skin done by the patient. The hormone insulin is a well-known injectable therapy. More recently studies showed that another hormone, glucagon-like peptide 1 (GLP-1), has a glucose-lowering effect similar to insulin. GLP-1-based therapies have several advantages such as low risk of too low glucose levels and increased body weight loss. We studied patients that switched from insulin therapy to liraglutide, a GLP-1-based injectable therapy. We found that after 1 year the majority of patients switching from insulin still continued liraglutide. We compared baseline characteristics between patients that continued liraglutide and patients that discontinued because glucose levels were too high. We found that patients with lower doses of insulin and less frequent use of insulin were more likely to continue liraglutide after 1 year. Second, we show that patients that reach their target levels of HbA1c, a measure of normal glucose levels over a longer time, have lower HbA1c at baseline and shorter durations of diabetes and insulin therapy. To conclude, we show patient characteristics that can be used in clinical practice to predict which patients on insulin can benefit from GLP-1-based therapy.

\section{INTRODUCTION}

GLP-1 receptor agonists (GLP-1 RA) are increasingly used in patients with type 2 diabetes mellitus (DM2). In patients inadequately controlled with oral antidiabetic medication, GLP-1 RA have demonstrated at least comparable efficacy to insulin [1]. For patients already on insulin, evidence and guidelines support addition of a GLP-1 RA as combinational injection therapy for better glycemic control [2]. However until recently, combining both injectables was not possible in our patients because of local regulation and reimbursement policies. Therefore, selected patients on insulin that wanted to benefit from the beneficial effects of GLP-1 RA such as ease of use, weight loss, and low risk of hypoglycemia were considered to be switched from insulin to GLP-1 RA. Limited evidence is available on the efficacy of a switch from insulin to a GLP-1 RA. In patients randomized to switching from insulin to the GLP-1 RA exenatide or maintaining their insulin regimen, exenatide alone was able to sustain glycemic control [3]. Short-term studies on an insulin-to-liraglutide switch in relatively lean Japanese patients using low doses of liraglutide and no concurrent treatment of metformin or sulfonylurea (SU) showed that $57-75 \%$ of the patients continued liraglutide with overall improvement in glycemic control [4-7]. Determinants of success were shorter duration of DM2, lower insulin dose, lower HbA1c levels, and higher C-peptide concentrations at baseline. However, the questions arises if these studies can be applied in our patients that as a result of reimbursement policy have a BMI $\geq 35 \mathrm{~kg} / \mathrm{m}^{2}$ and the GLP-1 RA should be combined with SU and metformin in the maximal tolerated dose.

In this study we retrospectively studied obese patients switching from insulin to the GLP-1 RA liraglutide with SU and metformin. We show the efficacy in this group and predictors of success. In the current age of combined use of GLP-1 RA and insulin, this study gives a unique insight into which patients may reduce insulin dose when starting add-on therapy with a GLP-1 RA. 


\section{METHODS}

In this retrospective study, all consecutive patients with DM2 undergoing an insulin-to-liraglutide switch during routine medical care at the outpatient clinic of OLVG Amsterdam (January 2009-February 2015) were included. The study was approved by the local ethics committee Adviescommissie Wetenschappelijk Onderzoek-Medisch-Ethische Commissie (ACWO-MEC) OLVG (ID WO 15.086) and was done in accordance with the Declaration of Helsinki. In accordance with the institutional protocol regarding a insulin-to-liraglutide switch, a random C-peptide was measured in all persons and a level of $>0.5 \mathrm{nmol} / \mathrm{l}$ was advised before discontinuation of insulin. Eligible patients discontinued insulin and on the following day commenced liraglutide treatment together with an SU. Metformin was prescribed earlier in a maximally tolerated dose. During this time the patient was in close contact with the diabetes nurse to monitor glucose values. Liraglutide was started at a dose of $0.6 \mathrm{mg} /$ day and increased if tolerated to a maximum of $3.6 \mathrm{mg} /$ day. The decision to replace insulin for liraglutide, dosing schedule for liraglutide, dosing schedule for SU, and continuation or discontinuation of liraglutide were made on an individual basis during routine medical care. For this retrospective analysis we defined success rate as continuation or discontinuation of liraglutide as decided by the treating physician at 12 months after replacing insulin for liraglutide. For patients that discontinued liraglutide it was registered if they discontinued because of side effects or discontinued because of poor glycemic control (Dglyc). Baseline characteristics were extracted from the medical records and compared between patients that continued liraglutide and Dglyc. A subgroup analysis was performed in patients that continued liraglutide with known $\mathrm{HbA} 1 \mathrm{c} \leq 7 \% \quad(53 \mathrm{mmol} / \mathrm{mol} ; \quad L$ $\leq 53)$ and $\mathrm{HbA} 1 \mathrm{c}>7 \%(53 \mathrm{mmol} / \mathrm{mol} ; L>53)$ at 12 months (or 9 months if not available). For the HbA1c and body weight time course, values were extracted at 3 months ( \pm 6 weeks), 6 months ( \pm 6 weeks), 9 months ( \pm 6 weeks), and 12 months ( \pm 6 weeks). SPSS (version 24) was used to conduct the statistical analyses.

\section{RESULTS}

During routine medical care 104 patients made an insulin-to-liraglutide switch (43\% male; mean age $57.2 \pm 9.9$ years; mean BMI $\left.39.8 \pm 5.4 \mathrm{~kg} / \mathrm{m}^{2}\right)$. Sixty patients still continued liraglutide after 12 months (58\%; Cont) whereas 37 patients discontinued treatment because of poor glycemic control within 12 months (36\%; Dglyc). Seven patients discontinued liraglutide because of intolerable side effects (7\%). Most patients received a final dose of $1.8 \mathrm{mg}$ of liraglutide (min-max 0.6-3.6 mg). In the Dglyc group, liraglutide was used during a minimum of 3 days and a maximum of 362 days, with an almost linear distribution of discontinuation throughout the year (Suppl Fig. 1). For persons that stopped because of intolerable side effects, six out of seven discontinued liraglutide in the first 120 days (Suppl Fig. 1). The intolerable side effects were classified as gastrointestinal (e.g., nausea, diarrhea, vomiting, abdominal pain) in five persons compared to other causes in two persons.

Baseline characteristics were compared between patients that continued and discontinued because of poor glycemic control. No differences in gender $(p=0.155)$, age $(p=0.600)$, and BMI $(p=0.938)$ at baseline were observed between the Cont and Dglyc groups. Insulin dose at baseline was significantly higher in patients that discontinued because of poor glycemic control (Dglyc $144 \pm 77$ versus Cont $86 \pm 49$ units per day; $p<0.001$; Fig. 1a). The percentages of patients who continued liraglutide were high in patients using a once daily or twice daily regimen (respectively $91 \%$ and $73 \%$ ) versus $38 \%$ of patients using a flexible (four times daily) regimen, showing a significant effect of insulin regimen on continuation of liraglutide $(p<0.001$; Fig. 2b). No significant difference in C-peptide concentrations was observed (Fig. 1c).

We performed a subgroup analysis of patients continuing liraglutide with a target HbA1c $(L \leq 53$; after treatment mean HbA1c 
a

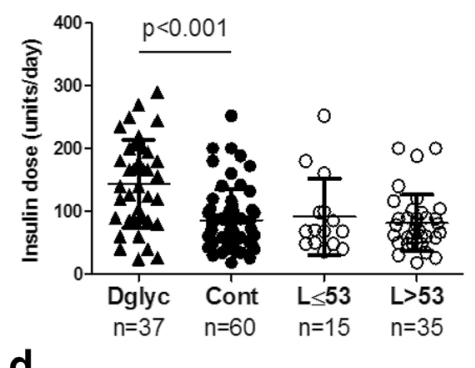

d

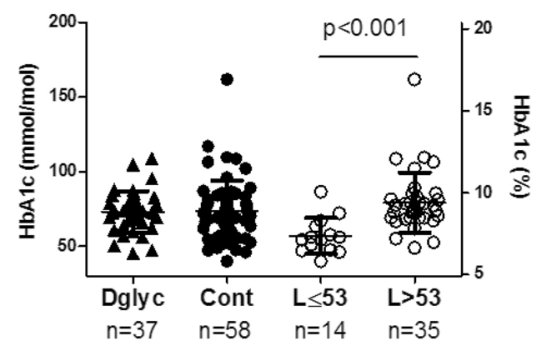

b
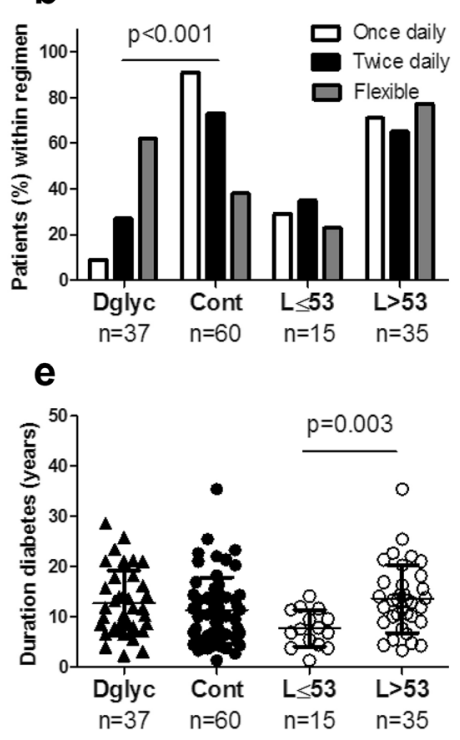

C

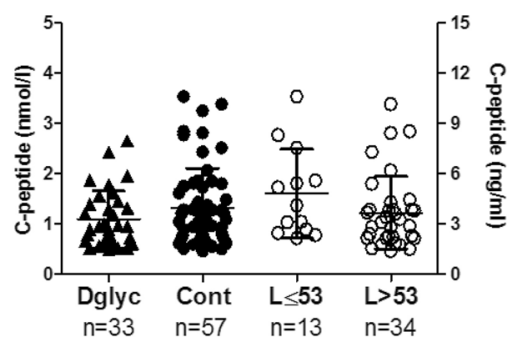

f

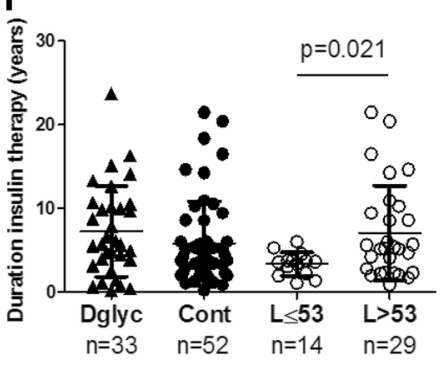

Fig. 1 Comparison of baseline characteristics between groups Dglyc (filled triangles; discontinuation due to glycemic deterioration) and Cont (filled circles; continued liraglutide) and subgroup analysis of patients reaching or maintaining $\mathrm{HbAlc} \leq 7 \%(53 \mathrm{mmol} / \mathrm{mol} ; L \leq 53)$ and patients with $\mathrm{HbAlc}>7 \%(53 \mathrm{mmol} / \mathrm{mol} ; \quad L>53)$ : a insulin dose, b patients (\%) within regimen (total

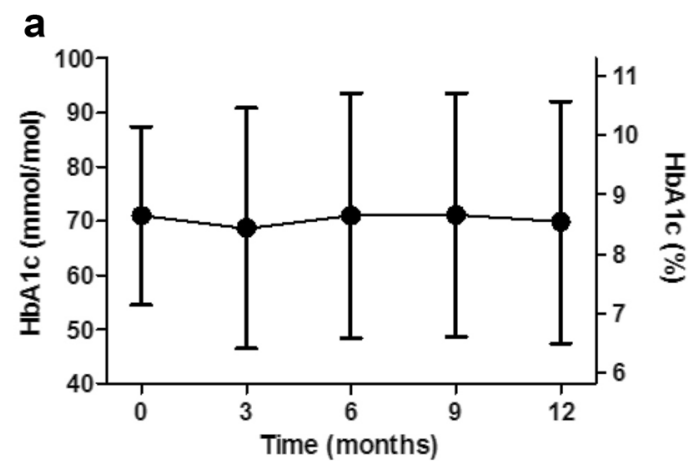

Fig. 2 a Time course of HbAlc for 52 patients that continued liraglutide for 12 months. b Time course of body weight for 50 patients who continued liraglutide for

$47 \pm 4 \mathrm{mmol} / \mathrm{mol}$ ) after a year and patients with an HbA1c above target $(L>53$; after treatment mean HbA1c $80 \pm 20 \mathrm{mmol} / \mathrm{mol}$ ). Baseline serum HbA1c levels were significantly lower in the patients who reached or maintained the target HbA1c on liraglutide $(L \leq 53$; patients once daily, $n=22$; twice daily, $n=33$; flexible, $n=42$ ), c C-peptide, d HbAlc, e duration diabetes, f duration insulin therapy. Continuous variables scatter of subjects and mean \pm SD. Grouped variable \% within group. $P$ value shown is for unpaired two-sided $t$ test for continuous variables and $\chi^{2}$ for insulin regimen between

Dglyc and Cont or $L \leq 53$ and $L>53$

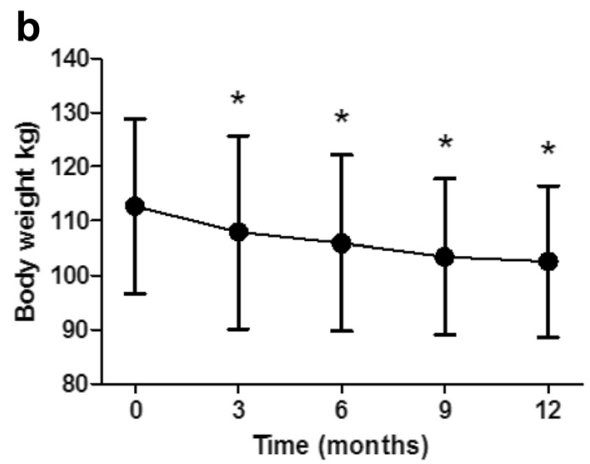

12 months, significant effect of repeated measurement GLM followed by post hoc test. ${ }^{*} p<0.05$ compared to baseline. Data shown in mean $\pm S D$

mean $57 \pm 12 \mathrm{mmol} / \mathrm{mol}(7.4 \pm 3.2 \%)$ versus $L>53$; mean $79 \pm 20 \mathrm{mmol} / \mathrm{mol}(9.4 \pm 4.0 \%)$; Fig. 1d). Mean duration of DM2 $(L \leq 53$, $7.7 \pm 3.6$ years; $\quad L>53, \quad 13.6 \pm 6.8$ years; Fig. 1e) and mean duration of insulin therapy $(L \leq 53,3.4 \pm 1.4$ years; $L>53,7.1 \pm 5.6$ years; 
Fig. 1f) were significantly less in the $L \leq 53$ group.

In patients that continued liraglutide, $\mathrm{HbA} 1 \mathrm{c}$ levels from 52 out of 60 patients and body weight from 50 out of 60 patients could be extracted from the medical records. On average there was no significant change in $\mathrm{HbA1c}$ in patients that continued liraglutide (Fig. 2a) with a significant decrease of body weight (Fig. 2b).

\section{DISCUSSION}

This is the first study evaluating an insulin-toliraglutide switch in obese patients with type 2 diabetes with the concurrent use of SU and metformin during routine medical care. We found that overall the majority of persons $(58 \%)$ continued liraglutide after 1 year with on average no change in HbA1c and decrease of body weight. Insulin dose and regimen were predictive of overall continuation of liraglutide, whereas baseline HbA1c levels, duration of diabetes, and duration of insulin therapy were predictive of reaching the target $\mathrm{HbA} 1 \mathrm{c}$ on liraglutide.

Our observation that the majority of patients continued liraglutide after 1 year with on average no improvement of glycemic control is in line with the 16-week study on an insulin-toexenatide switch [3]. Furthermore, patients with insufficient glycemic control on GLP-1 RA did not improve glycemic control when switching to insulin alone [8]. In contrast, Japanese studies in relatively lean patients have shown similar continuation rates $(57-75 \%)$ but improvements of HbA1c levels were observed after switching to liraglutide [4-6]. Our study in obese patients supports the current guidelines that for patients already on insulin, switching to liraglutide alone on average does not improve glycemic control and could cause glycemic detoriation.

For patients with good glycemic control on insulin, however, one could consider switching to GLP-1 RA because of ease of use, low risk of hypoglycemia, weight loss, and more recently decreased cardiovascular risk and NAFLD $[9,10]$. One could start a GLP-1 RA on top of insulin and subsequently reduce insulin dose if possible. Our study indicates which patients are most likely to reach glycemic targets on liraglutide alone. In this study no patient with an HbA1c above $87 \mathrm{mmol} / \mathrm{mol}$, diabetes duration above 15 years, and duration of insulin therapy above 6 years reached the glycemic target on liraglutide alone. Disease duration and low baseline HbA1c have been shown previously to predict good glycemic control on a GLP-1 RA both after an insulin-toexenatide switch and after oral antidiabetic medication $[3,11,12]$. In contrast, we did not observe higher levels of C-peptide, a marker of remaining beta cell capacity, in patients that successfully switched to liraglutide as previously described [3-7, 11, 12]. However, it should be noted that based on previous studies our institutional guidelines prescribe that all patients considered for a switch need to have C-peptide levels $>0.50 \mathrm{nmol} / \mathrm{l} \quad(1.50 \mathrm{ng} / \mathrm{ml}) \quad$ omitting patients with low C-peptide levels. Furthermore, our C-peptide values were not standardized for meal or time of day, causing significant variation. A recent study showed also no relation between response to liraglutide or dulaglutide and fasting C-peptide, whereas previously postprandial C-peptide has been shown to be a better indicator of success $[6,7,13]$.

There were several limitations of this study. As a result of the retrospective nature of this study no criteria for the selection of patients, other than a $\mathrm{BMI} \geq 35 \mathrm{~kg} / \mathrm{m}^{2}$ and C-peptide levels $>0.5 \mathrm{nmol} / \mathrm{l}$, were set. Also no predefined guidelines for dosing of oral antidiabetic agents (metformin/SU)/liraglutide and discontinuation of liraglutide were established. This represents clinical practice as these decisions are made for every patient individually on the basis of several factors. Even in this real-life setting we found similar rates of continuation as a previous study using predefined glycemic targets [3]. In spite of these shortcomings we believe this paper gives new insight into the efficacy and determinants of success on liraglutide.

\section{CONCLUSIONS}

We show that the majority of obese patients continued liraglutide with SU and metformin for at least 1 year after stopping insulin with on average no change in glycemic control and body 
weight reduction. This study shows predictors of success, which can be studied prospectively to indicate which patients on insulin could make a successful switch to liraglutide with rapid reduction of insulin dosage.

\section{ACKNOWLEDGEMENTS}

We thank all patients in this study, and the diabetes team, Rosa Regez and Ed Slaats for patient and research support.

Funding. No funding or sponsorship was received for this study or publication of this article. The article processing charges were funded by Stichting Wetenschap en Onderzoek Interne Geneeskunde OLVG. All authors had full access to all of the data in this study and take complete responsibility for the integrity of the data and accuracy of the data analysis.

Authorship. All named authors meet the International Committee of Medical Journal Editors (ICMJE) criteria for authorship for this article, take responsibility for the integrity of the work as a whole, and have given their approval for this version to be published.

Disclosures. All authors (E. Bruinstroop, L. Meyer, C.B. Brouwer, D.E. van Rooijen, and P.S. van Dam) have nothing to disclose.

Compliance with Ethics Guidelines. All procedures performed in studies involving human participants were in accordance with the ethical standards of the institutional and/or national research committee and with the 1964 Declaration of Helsinki and its later amendments or comparable ethical standards. This research used nonidentifiable data obtained by the treating physicians and therefore on the basis of the decision from our local ethics committee Adviescommissie Wetenschappelijk Onderzoek-Medisch-Ethische Commissie (ACWO-MEC) OLVG (ID WO 15.086) informed consent was not required. Patients have had the opportunity to object to the use of their data for retrospective scientific research, but none of the patients did.
Data Availability. The data sets analyzed during the current study are available from the corresponding author on reasonable request.

Open Access. This article is distributed under the terms of the Creative Commons AttributionNonCommercial 4.0 International License (http:// creativecommons.org/licenses/by-nc/4.0/), which permits any noncommercial use, distribution, and reproduction in any medium, provided you give appropriate credit to the original author(s) and the source, provide a link to the Creative Commons license, and indicate if changes were made.

\section{REFERENCES}

1. Abd El Aziz MS, Kahle M, Meier JJ, Nauck MA. A meta-analysis comparing clinical effects of short- or long-acting GLP-1 receptor agonists versus insulin treatment from head-to-head studies in type 2 diabetic patients. Diabetes Obes Metab. 2017;19:216-27.

2. Eng C, Kramer CK, Zinman B, Retnakaran R. Glucagon-like peptide-1 receptor agonist and basal insulin combination treatment for the management of type 2 diabetes: a systematic review and meta-analysis. Lancet. 2014;384:2228-34.

3. Davis SN, Johns D, Maggs D, Xu H, Northrup JH, Brodows RG. Exploring the substitution of exenatide for insulin in persons with type 2 diabetes treated with insulin in combination with oral antidiabetes agents. Diabetes Care. 2007;30:2767-72.

4. Usui $\mathrm{R}$, Yabe $\mathrm{D}$, Kuwata $\mathrm{H}$, et al. Retrospective analysis of safety and efficacy of insulin-to-liraglutide switch in Japanese type 2 diabetes: a caution against inappropriate use in persons with reduced $\beta$-cell function. J Diabetes Investig. 2001;4:585-94.

5. Kawata T, Kanamori A, Kubota A, et al. Is a switch from insulin therapy to liraglutide possible in Japanese type 2 diabetes mellitus persons? J Clin Med Res. 2014;6:138-44.

6. Iwao T, Sakai K, Sata M. Postprandial serum C-peptide is a useful parameter in the prediction of successful switching to liraglutide monotherapy from complex insulin therapy in Japanese persons with type 2 diabetes. J Diabetes Complicat. 2013;27:87-91.

7. Araki H, Tanaka Y, Yoshida S, et al. Oral glucosestimulated serum C-peptide predicts successful 
switching from insulin therapy to liraglutide monotherapy in Japanese persons with type 2 diabetes and renal impairment. J Diabetes Investig. 2014;5:435-41.

8. Montvida O, Klein K, Kumar S, Khunti K, Paul SK. Addition of or switch to insulin therapy in people treated with glucagon-like peptide- 1 receptor agonists: a real-world study in 66583 patients. Diabetes Obes Metab. 2017;19:108-17.

9. Marso SP, Daniels GH, Brown-Frandsen K, et al. Liraglutide and cardiovascular outcomes in type 2 diabetes. N Engl J Med. 2016;375:311-22.

10. Armstrong MJ, Gaunt P, Aithal GP, et al. Liraglutide safety and efficacy in patients with non-alcoholic steatohepatitis (LEAN): a multicentre, double-blind, randomised, placebo-controlled phase 2 study. Lancet. 2016;387:679-90.

11. Jones AG, Shields BM, Hyde CJ, Henley WE, Hattersley AT. Identifying good responders to glucose lowering therapy in type 2 diabetes: implications for stratified medicine. PLoS One. 2014;9:e111235.

12. Jones AG, McDonald TJ, Shields BM, et al. Markers of $\beta$-cell failure predict poor glycemic response to GLP-1 receptor agonist therapy in type 2 diabetes. Diabetes Care. 2016;39:250-7.

13. Iwamoto N, Matsui A, Kazama H, Oura T. Subgroup analysis stratified by baseline pancreatic $\beta$-cell function in a Japanese study of dulaglutide in patients with type 2 diabetes. Diabetes Ther. 2018;9:383-94. 